\title{
Is Quorum Sensing Interference a Viable Alternative to Treat Pseudomonas aeruginosa Infections?
}

\author{
Rodolfo García-Contreras * \\ Department of Microbiology and Parasitology, Faculty of Medicine, National Autonomous University of Mexico, Mexico City, \\ Mexico
}

Quorum sensing (QS) coordinates the expression of multiple virulence factors in Pseudomonas aeruginosa; hence its inhibition has been postulated as a new alternative to treat its infections. In particular, QS interference approaches claim that they attenuate bacterial virulence without directly decreasing bacterial growth and suggest that in vivo the immune system would control the infections. Moreover, since in vitro experiments performed in rich medium demonstrate that interfering with QS decreases the production

OPEN ACCESS

Edited by:

Axel Cloeckaert,

French National Institute for Agricultural Research (INRA), France

Reviewed by:

Pierre Cornelis,

Vrije Universiteit Brussel, Belgium César De La Fuente-Núñez,

Massachusetts Institute of Technology, USA

*Correspondence: Rodolfo García-Contreras

rgarc@bq.unam.mx

Specialty section:

This article was submitted to

Antimicrobials, Resistance and Chemotherapy,

a section of the journal

Frontiers in Microbiology

Received: 01 June 2016

Accepted: 31 August 2016

Published: 14 September 2016

Citation:

García-Contreras R (2016) Is Quorum

Sensing Interference a Viable

Alternative to Treat Pseudomonas

aeruginosa Infections?

Front. Microbiol. 7:1454.

doi: 10.3389/fmicb.2016.01454 of virulence factors without affecting bacterial growth it was assumed than in vivo therapies will minimize the selection of resistant strains. Therefore, the underlying assumptions toward an effective implementation of a successful Quorum sensing interference (QSI) therapy for treating P. aeruginosa infections are that (i) QS only exerts important effects in the regulation of virulence genes but it does not affect metabolic processes linked to growth, (ii) the expression of virulence factors is only positively regulated by QS, (iii) inhibition of virulence factors in vivo do not affect bacterial growth, (iv) the immune system of the infected patients will be able to get rid of the infections, and (v) the therapy will be effective in the strains that are actively producing the infections. Nevertheless, for QSI in P. aeruginosa, substantial experimental evidence against the validity of most of these assumptions has accumulated during the past years, suggesting that a far better understanding of its virulence and its behavior during infections is needed in order to design truly solid QSI therapeutic alternatives to combat this remarkable pathogen.

Keywords: Pseudomonas infections, quorum sensing (QS), quorum quenching, resistance, clinical isolates

\section{INTRODUCTION}

Pseudomonas aeruginosa is a remarkable opportunistic pathogen that infects patients that are immunocompromised, have severe burns, cancer, or AIDS, are intubated and with prosthetic devices, and also those suffering from chronic affections like cystic fibrosis (CF). This bacterium is a major health problem worldwide being responsible of $10 \%$ of nosocomial infections (Antunes et al., 2010; Castillo-Juárez et al., 2015), since it is intrinsically resistant to several antimicrobials (Poole, 2011) and able to develop resistance against new ones, has a high biofilm production and produces an arsenal of virulence factors. One of the main mechanisms that controls the production of its virulence factors is quorum sensing (QS) which coordinates the expression of such factors 
once bacteria have reached a high population density, thus maximizing their chances to overcome the host defenses and establish the infection.

QS systems are common in bacterial pathogens; among Gram negatives, in addition to P. aeruginosa, Acinetobacter baumannii (Bhargava et al., 2010), Escherichia coli (Sperandio et al., 2002) Salmonella strains (Choi et al., 2007), and Vibrio strains (Zhu et al., 2002; Yang and Defoirdt, 2015) use them to coordinate their virulence. Hence, QS interference (QSI) or quorum quenching (QQ) is a strategy proposed to inhibit virulence as an alternative to treat the infections of several important bacterial pathogens (Castillo-Juárez et al., 2015).

In this opinion piece I focus in $P$. aeruginosa, one of the most studied organisms regarding QS and QSI, nevertheless what is exposed here may be also applicable to other bacterial pathogens.

$P$. aeruginosa is one of the more complex known bacterial pathogens; it possesses an ample genome and a high percentage of its genes are devoted to gene regulation (Stover et al., 2000). Regarding QS systems, it has a hierarchical architecture governed by the LasRI module which produces and senses $\mathrm{N}$-3-oxododecanoyl-L-Homoserine lactone and activates the expression of multiple virulence genes such as those producing elastase $\mathrm{A}$ and $\mathrm{B}$, pyocyanin, alkaline and protease, and activating a second homoserine lactone (HSL) QS module known as RhlRI, which produces and senses N-butyryl-L-Homoserine lactone. RhlR bound to its autoinducer also activates directly some virulence genes like those encoding rhamnolipids and pyocyanin (Smith and Iglewski, 2003; Jimenez et al., 2012). In addition, P. aeruginosa also has a quinolone dependent system known as PQS, which is positively regulated by LasR and negatively by RhlR, forming a complex and intricate network (Jimenez et al., 2012; Lee and Zhang, 2015). In addition to the QS intrinsic components, several regulators such as GacA/GacS, QscR, Vfr, RpoN, and RpoS, influence the expression of QS dependent factors (Lee and Zhang, 2015).

The complex relationship between QS and virulence in $P$. aeruginosa had been recently evidenced by the fact that las $R$ deficient mutants (including clinical strains) growing at slow rates or in the stationary phase, overproduce pyocyanin due a lack of repression of the phenazine genes by RsaL, a negative transcriptional regulator positively controlled by LasR (Cabeen, 2014). Moreover, in lasR mutants, the activity of the $r h l$ system is only delayed but not abolished, allowing significant production of pyocyanin, rhamnolipids and N-butyryl-L-Homoserine lactone (which are RhlR dependent) and even the production of the LasR dependent QS signals N-3-oxo-dodecanoyl-L-Homoserine lactone and PQS (Dekimpe and Deziel, 2009). These findings suggest that the inhibition of a particular component of the QS network, even the pivotal factor LasR, may be counteracted by the activation of alternative components of the network. Indeed, some recent studies have identified novel QS inhibitors for which RhlR and not LasR as the relevant in vivo target (O'Loughlin et al., 2013; Welsh et al., 2015).

In addition, recently it was discovered that an environmental strain (148 isolated from dolphin gastric juice) produces significant amounts of the QS-controlled virulence factors rhamnolipids and pyocyanin. Critically, this strain is virulent to mice, even without having a las $R$ gene and without producing N-3-oxo-dodecanoyl-L-Homoserine lactone. Hence, in this strain, the control of QS dependent virulence factors must be exerted by other regulators (Grosso-Becerra et al., 2014).

QS Interference could be achieved by the attenuation of the QS communication systems via: (i) the disruption of the QS receptors, (ii) the degradation of the autoinducers signals or (iii) the inhibition of the signal synthesis. Accordingly, several QS inhibitors or quorum quenchers (QQ) suitable for $P$. aeruginosa have been developed, under the assumptions that (i) the expression of virulence factors is only positively regulated by QS, (ii) the QQ will only exert significant effects in the regulation of virulence genes but not in metabolic processes linked to growth, hence avoiding or at least decreasing the generation of selective pressure that leads to resistance, (iii) the immune system of the infected patients will be able to get rid of the infections, and (iv) the therapy will be effective against the strains that are actively producing the infections. Nevertheless, substantial evidence against the validity of those assumptions has accumulated over the years.

\section{QUORUM SENSING INFLUENCES METABOLIC PROCESSES AND BACTERIAL GROWTH}

Although the role of QS in P. aeruginosa virulence has been extensively studied, whether it influences global metabolism and cell growth is less explored. One possible reason for this bias is that at the transcriptomic level, few significant changes in metabolicrelated genes are found when QS proficient and deficient strains are compared or when QS inhibitors are administrated to QS proficient strains (Hentzer et al., 2003; Schuster et al., 2003; Wagner et al., 2003). Nevertheless, recently Davenport and coworkers found that the metabolome of a lasI rhlI double mutant (unable to produce HSL QS autoinducers) and that of its wild-type progenitor have a notorious divergence once the wild-type strain produced the highest levels of autoinducers; remarkably, around one third of all the metabolites identified changed (Davenport et al., 2015). This phenomenon is understandable since the wild-type strain devotes many of its resources to the production of costly virulence factors such as exoproteases, phenazines, and exopolysacharides, while, in contrast, the QS mutants uses the same resources for cell division. In agreement, the QS deficient mutants achieve higher growth yields than QS wild-type strains (Diggle et al., 2007).

Although the possible implications of the metabolic divergence in QS mutants on bacterial physiology and QS interference therapies is still unexplored, Davenport and coworkers demonstrated that important metabolic changes in the membrane metabolism of the wild-type strain are driven by QS upon entering the stationary phase. These modifications include increased fatty acid saturation, chain length, and cyclopropanation, which in turn, promote the generation of robust cell membranes. In contrast, the membranes of QS mutants do not have these modifications and are therefore more susceptible to stress. 
In addition to these metabolic changes, several works have demonstrated that in $P$. aeruginosa and other bacteria, QS enhances the stress response (Bjarnsholt et al., 2005; Bhargava et al., 2014; de Oca-Mejia et al., 2015; García-Contreras et al., 2015a); for example, by upregulating antioxidant enzymes such as catalase, superoxide dismutase (Hassett et al., 1999) and NADPH-generating enzymes (García-Contreras et al., 2015a). This is important since during an infection, the immune system attacks bacteria by releasing reactive oxygen species, and at least in vitro oxidative stress is able to select functional QS systems as well as QS interference resistant mutants (García-Contreras et al., 2015a).

Moreover, growth of $P$. aeruginosa can be strongly dependent on QS either if adenosine or protein is used as sole carbon sources, since nuh, the gene encoding the nucleoside hydrolase, is under tight LasR control (Heurlier et al., 2005) and since the expression of exoproteases is under QS control (Diggle et al., 2007). During infections, adenosine utilization (Patel et al., 2007; Sheng et al., 2012) as well as the degradation of host proteins (Wretlind and Pavlovskis, 1983; Laarman et al., 2013) are important for bacterial virulence and survival.

All the above summarized facts demonstrate that QS is an important regulator of bacterial metabolism and physiology and suggest that QS interference will have the direct effect of decreasing bacterial growth and viability in vivo. In agreement, several in vivo infection studies have shown that when a QS interference therapy is able to increase animal survival (and decrease damage to the host), there is a significant decrease in bacterial counts in the infection sites (Wu et al., 2004; Christensen et al., 2007; Defoirdt et al., 2010; Jakobsen et al., 2011). The decrease in bacterial viability due QS interference may be an important potential source of in vivo selective pressure for the selection of bacterial resistance (García-Contreras et al., 2016).

\section{POTENTIAL INCREASE IN VIRULENCE UPON QUORUM SENSING INTERFERENCE}

Although the production of multiple virulence factors in $P$. aeruginosa is positively regulated by $\mathrm{QS}$, the virulence phenotype displayed by this organism is a complex combinatorial phenomenon that cannot be easily predicted with the identification of the presence or absence of a set of specific genes (Lee et al., 2006; Grosso-Becerra et al., 2014). In agreement, several studies had shown that clinical strains display a wide variety levels of virulence (Fenner et al., 2006; Lee et al., 2006; Garcia-Contreras et al., 2015b) and that environmental strains often conserve high virulence (Grosso-Becerra et al., 2014).

An overlooked fact about the role of QS in P. aeruginosa virulence was discovered in 2005 by Bleves and coworkers, who that found that in contrast to several other virulence traits, the expression of the type III secretion system (TTSS) in $P$. aeruginosa is negatively regulated by QS, specifically by RhlR and PqsR (Bleves et al., 2005; Kong et al., 2009). An important factor in such negative regulation of virulence genes by QS is that it requires low calcium levels, and hence several transcriptomics studies done in culture medium such as LB with relative high calcium levels fail to show this relationship. Since the TTSS is an important determinant of virulence in several animal infection models including pneumonia, peritonitis, bacteremia, burn infections, and keratitis (Hauser, 2009), the possibility that QS interfering therapies may be activating this system and therefore promoting virulence should not be ignored. Moreover, $P$. aeruginosa has five of the six known types of secretion systems present in Gram negative bacteria (all but type IV) and some of these systems, like type VI, are present in several copies in its genome (Bleves et al., 2010). The QS influence on the expression of these secretion systems is as of yet unknown, with the exceptions of the TTSS and the second type VI secretion system that are negatively regulated by QS (Sana et al., 2012).

More striking is the fact that QS interference using azithromycin selects the wild-type virulent phenotype against the less virulent lasR mutants in intubated patients colonized by $P$. aeruginosa (Kohler et al., 2010). This surprising fact is understandable since lasR mutants often appear and are selected in infections since they act as phenotypic cheaters that utilize the public goods such as exoproteases and siderophores produced by the cooperative wild-type individuals (Diggle et al., 2007; Sandoz et al., 2007). Hence, inhibiting QS removes the advantage of the lasR mutants and thereby selects the wild-type, thus a detrimental effect of QS interference in the long run could be to increase the prevalence of virulent genotypes in the nosocomial environment (Kohler et al., 2010).

Another key aspect of current QS inhibitors like furanones is that, depending on their concentration, they can activate rather than inhibit QS (Martinelli et al., 2004). Furthermore, related QS inhibitors such as synthetic HSLs can activate rather than inhibit some virulence factors (Welsh et al., 2015).

\section{LIMITATIONS OF THE CURRENT ANIMAL INFECTION MODELS FOR THE STUDY OF QUORUM SENSING INTERFERENCE}

To date, several animal infection models have demonstrated that QS deficient mutants are much less virulent than their QS proficient parental strains; accordingly, QS interference promotes an increase in host survival and a decrease in damage and bacterial counts (Castillo-Juárez et al., 2015). The infection models used include arthropods like Galleria mellonela, nematodes such as Caenorhabditis elegans, fruit fly, and zebrafish which are valuable and informative but that do not accurately reflect human physiology. Moreover, when mice are used as a model, immune competent individuals are evaluated; hence, the fact that $P$. aeruginosa is a strict opportunistic pathogen that does not attack individuals with competent immune systems is overlooked, and so it is not clear if the immune systems of immunosuppressed patients will be able to clear the bacteria from infections upon QS interference treatments.

In addition to attacking immunosupressed individuals, $P$. aeruginosa is the major cause of death of CF patients; accordingly, there are several mice models that incorporate 
different mutations in the CF transmembrane conductance regulator (CTFR) protein and that are able to mimic some of the characteristics of the disease in humans. However, CF is a very complex disease and there are more than one thousand reported mutations in the cftr gene associated with the disease; hence, developing a murine model that closely resembles the human disease is challenging (Guilbault et al., 2007).

Most of the current models are suitable to study acute infections and only a few like the one developed in 2005 by Hoffmann and coworkers are optimized to simulate chronic CF infections (Hoffmann et al., 2005). Importantly, this model has been used to demonstrate that QS interference with azithromycin inhibits alginate production of the mucoid NH57388A strain in vivo, attenuating the damage produced to the host, but unfortunately it was not able to significantly increase mouse survival (Hoffmann et al., 2007).

Clinical studies have demonstrated that AZM treatment improves lung function in CF patients (Saiman et al., 2003); however, besides the QSI effect, azithromycin has bactericidal and anti-inflammatory effects; hence, the improvement of both mice and patients is likely due a combination of these effects rather than an exclusive consequence of the QSI properties of azithromycin. Therefore, testing of the effect of more specific QS interference molecules in these kind of models is needed in order to elucidate the potential of these therapies for CF patients.

Another kind of mouse model that more closely resembles the situation observed in humans is the thermally-induced injury model, which consists of producing a burn of second or third degree on the dorsal side of the mouse using hot water and subsequent inoculation of the burn. The utilization of this model has confirmed that QS-deficient mutants such as lasR, lasI, rhlI, lasI rhlI, and pqsA (Rumbaugh et al., 1999a,b; Lesic et al., 2007), have less virulence than the parental strains. Moreover, it has been used to test the effect of the halogenated anthranilic acid analogs $6 \mathrm{FABA}, 6 \mathrm{CABA}$, and $4 \mathrm{CABA}$ which are inhibitors of the synthesis of quinolone QS signals. These compounds, at the doses administrated, decrease mouse mortality significantly. Nevertheless, they were tested at a single dose (Lesic et al., 2007), so it is unknown if they exert dose response effects. This is not trivial since as mentioned before, QS inhibitors may act as QS activators depending on the used doses (Martinelli et al., 2004).

\section{INSENSIBILITY OF SOME CLINICAL STRAINS TO CURRENT QUORUM QUENCHERS}

Before 2010 it was assumed than QS interference will be impervious or at least less susceptible to promote bacterial resistance than conventional antibiotic therapies (Defoirdt et al., 2010). In 2011, it was demonstrated that $P$. aeruginosa acquires resistance easily against the canonical HSL-dependant quorum quencher furanone C-30, by activating the multidrug efflux pump MexAB-OprM (Maeda et al., 2012). The activation of this pump is mediated by mutations disrupting the transcriptional repressors MexR and NalC, and such mutations are common in clinical isolates, presumably since they are selected by intense antibiotic treatments (Tomas et al., 2010). As expected, these clinical isolates are resistant against C-30 (Maeda et al., 2012).

Recent studies have demonstrated that C-30 resistance is common in multidrug resistant strains. Importantly, resistance against other QQ compounds like 5-fluorouracil is also present in some clinical isolates and some antibiotic sensitive strains are also resistant to $\mathrm{C}-30$ by a decrease in the compound uptake. More strikingly, some of these strains produce higher amounts of the virulence factors in the presence of the furanone than in basal conditions (García-Contreras et al., 2013a,b; Garcia-Contreras et al., 2015b).

This suggests that if eventually QSI therapies are implemented in the clinic, they likely would not be effective against all the strains present in infections (García-Contreras et al., 2013a; Kalia et al., 2013), and that crucially they may fail to inhibit those strains with active antibiotic efflux pumps. Indeed, it was recently proposed that bacteria resistant to several QS inhibitors may be selected by QSI treatments (Koul et al., 2016).

\section{PERSPECTIVES}

Since recent evidence has demonstrated that QS is linked to basal metabolism and growth, it will be important to evaluate QS interference and its effect in bacteria growing in medium that more closely simulates the composition of the environment during infection (Palmer et al., 2007; GarciaContreras et al., 2015b), with attention on key metabolites that influence virulence and QS such as iron (Sokol and Woods, 1984; Mittal et al., 2008; Hazan et al., 2010), calcium (Sarkisova et al., 2005), phosphate (Zaborin et al., 2009), and adenosine (Patel et al., 2007; Sheng et al., 2012). Further, metabolomic and proteomic studies may shed light about the role of QS in bacterial physiology under in vivo-like conditions. In addition, the utilization of new technologies like microfluidics such as cell culture chips (organ-on-a-chip) would enable the interrogation of the roles of signaling, social cheating, mass transfer, and spatial organization in well-defined geometries, which is virtually impossible to asses at this moment using animal models.

Importantly, additional research about the ways $P$. aeruginosa may achieve resistance against QQ is needed in order to generate effective combination therapies less prone to the selection of resistance. One possibility is to exploit the fact that QS interference renders $P$. aeruginosa more sensitive to stress, including antibiotics and the effects of the immune system; hence, it is attractive to test if the combination of QS interference, antibiotic therapies and immunotherapy to improve the outcome of the therapies.

Another area of research that should be encouraged is the role of QS independent virulence factors and those negatively regulated by QS such as TTSS in infections and the effect of QS interference on these virulence determinants. Also, study of QS networks and the-virulence of environmental and clinical strains is needed since current evidence indicates these are highly variable (Grosso-Becerra et al., 2014; Garcia-Contreras et al., 
2015b) and that a specific QQ compound will not always be effective against all clinical strains. Also, more effort should be devoted to the study of the implementation of these therapies, since most of them are able to attenuate infections when the QQ compound is administrated shortly before the inoculation (having possible prophylactic effects), but the value of such therapies as a possible cure for an established infection should be better addressed.

Taking the available evidence together, it seems QS interference may be a valuable tool to combat $P$. aeruginosa infections; however, using QS inhibition as the sole therapy may not be an efficient strategy due several potential drawbacks. Hence, before QSI applications are used in the clinic, it is advisable to improve our current understanding of $P$. aeruginosa virulence with an emphasis on clinical strains and also on further defining the roles of QS in the physiology of this remarkable complex pathogen.

\section{REFERENCES}

Antunes, L. C., Ferreira, R. B., Buckner, M. M., and Finlay, B. B. (2010). Quorum sensing in bacterial virulence. Microbiology 156, 2271-2282. doi: 10.1099/mic.0.038794-0

Bhargava, N., Sharma, P., and Capalash, N. (2010). Quorum sensing in Acinetobacter: an emerging pathogen. Crit. Rev. Microbiol. 36, 349-360. doi: 10.3109/1040841X.2010.512269

Bhargava, N., Sharma, P., and Capalash, N. (2014). Pyocyanin stimulates quorum sensing-mediated tolerance to oxidative stress and increases persister cell populations in Acinetobacter baumannii. Infect. Immun. 82, 3417-3425. doi: 10.1128/IAI.01600-14

Bjarnsholt, T., Jensen, P. Ø., Burmølle, M., Hentzer, M., Haagensen, J. A., Hougen, H. P., et al. (2005). Pseudomonas aeruginosa tolerance to tobramycin, hydrogen peroxide and polymorphonuclear leukocytes is quorum-sensing dependent. Microbiology 151, 373-383. doi: 10.1099/mic.0.27463-0

Bleves, S., Soscia, C., Nogueira-Orlandi, P., Lazdunski, A., and Filloux, A. (2005). Quorum sensing negatively controls type III secretion regulon expression in Pseudomonas aeruginosa PAO1. J. Bacteriol. 187, 3898-3902. doi: 10.1128/JB.187.11.3898-3902.2005

Bleves, S., Viarre, V., Salacha, R., Michel, G. P., Filloux, A., and Voulhoux, R. (2010). Protein secretion systems in Pseudomonas aeruginosa: a wealth of pathogenic weapons. Int. J. Med. Microbiol. 300, 534-543. doi: 10.1016/j.ijmm. 2010.08.005

Cabeen, M. T. (2014). Stationary phase-specific virulence factor overproduction by a lasR mutant of Pseudomonas aeruginosa. PLoS ONE 9:e88743. doi: 10.1371/ journal.pone.0088743

Castillo-Juárez, I., Maeda, T., Mandujano-Tinoco, E. A., Tomas, M., Párez-Eretza, B., Garcia-Contreras, S. J., et al. (2015). Role of quorum sensing in bacterial infections. World J. Clin. Cases 3, 575-598. doi: 10.12998/wjcc.v3.i7.575

Choi, J., Shin, D., and Ryu, S. (2007). Implication of quorum sensing in Salmonella enterica serovar typhimurium virulence: the luxS gene is necessary for expression of genes in pathogenicity island 1. Infect. Immun. 75, 4885-4890. doi: 10.1128/IAI.01942-06

Christensen, L. D., Moser, C., Jensen, P. Ø., Rasmussen, T. B., Christophersen, L., Kjelleberg, S., et al. (2007). Impact of Pseudomonas aeruginosa quorum sensing on biofilm persistence in an in vivo intraperitoneal foreign-body infection model. Microbiology 153, 2312-2320. doi: 10.1099/mic.0.2007/006122-0

Davenport, P. W., Griffin, J. L., and Welch, M. (2015). Quorum sensing is accompanied by global metabolic changes in the opportunistic human pathogen Pseudomonas aeruginosa. J. Bacteriol. 197, 2072-2082. doi: 10.1128/ JB.02557-14

Defoirdt, T., Boon, N., and Bossier, P. (2010). Can bacteria evolve resistance to quorum sensing disruption? PLoS Pathog. 6:e1000989. doi: 10.1371/journal. ppat. 1000989

\section{AUTHOR CONTRIBUTIONS}

The author confirms being the sole contributor of this work and approved it for publication.

\section{FUNDING}

This work was supported by grants from SEP/CONACyT-México No. 152794, CONACYT Problemas Nacionales 2015 No. 201501-402 and PAPIIT-UNAM No. IA201116.

\section{ACKNOWLEDGMENTS}

I am grateful for critical reading of the manuscript and the valuable suggestions provided by Professor Gloria SoberónChávez from the Biomedical Science Institute at UNAM, Mexico and by Professor Thomas K. Wood from Penn State University.

Dekimpe, V., and Deziel, E. (2009). Revisiting the quorum-sensing hierarchy in Pseudomonas aeruginosa: the transcriptional regulator RhlR regulates LasRspecific factors. Microbiology 155, 712-723. doi: 10.1099/mic.0.022764-0

de Oca-Mejia, M. M., Castillo-Juarez, I., Martínez-Vázquez, M., Soto-Hernandez, M., and García-Contreras, R. (2015). Influence of quorum sensing in multiple phenotypes of the bacterial pathogen Chromobacterium violaceum. Pathog. Dis. 73, 1-4. doi: 10.1093/femspd/ftu019

Diggle, S. P., Griffin, A. S., Campbell, G. S., and West, S. A. (2007). Cooperation and conflict in quorum-sensing bacterial populations. Nature 450, 411-414. doi: 10.1038/nature06279

Fenner, L., Richet, H., Raoult, D., Papazian, L., Martin, C., and La Scola, B. (2006) Are clinical isolates of Pseudomonas aeruginosa more virulent than hospital environmental isolates in amebal co-culture test? Crit. Care Med. 34, 823-828. doi: 10.1097/01.CCM.0000201878.51343.F1

García-Contreras, R., Maeda, T., and Wood, T. K. (2013a). Resistance to quorumquenching compounds. Appl. Environ. Microbiol. 79, 6840-6846. doi: 10.1128/ AEM.02378-13

García-Contreras, R., Maeda, T., and Wood, T. K. (2016). Can resistance against quorum-sensing interference be selected? ISME J. 10, 4-10. doi: 10.1038/ismej. 2015.84

García-Contreras, R., Martinez-Vazquez, M., Velazquez Guadarrama, N., Villegas Paneda, A. G., Hashimoto, T., Maeda, T., et al. (2013b). Resistance to the quorum-quenching compounds brominated furanone C-30 and 5-fluorouracil in Pseudomonas aeruginosa clinical isolates. Pathog. Dis. 68, 8-11. doi: 10.1111/ 2049-632X.12039

García-Contreras, R., Nunez-López, L., Jasso-Chávez, R., Kwan, B. W., Belmont, J. A., Rangel-Vega, A., et al. (2015a). Quorum sensing enhancement of the stress response promotes resistance to quorum quenching and prevents social cheating. ISME J. 9, 115-125. doi: 10.1038/ismej.2014.98

Garcia-Contreras, R., Perez-Eretza, B., Jasso-Chavez, R., Lira-Silva, E., RoldanSanchez, J. A., Gonzalez-Valdez, A., et al. (2015b). High variability in quorum quenching and growth inhibition by furanone C-30 in Pseudomonas aeruginosa clinical isolates from cystic fibrosis patients. Pathog. Dis. 73:ftv040. doi: 10.1093/femspd/ftv040

Grosso-Becerra, M. V., Santos-Medellin, C., Gonzalez-Valdez, A., Mendez, J. L., Delgado, G., Morales-Espinosa, R., et al. (2014). Pseudomonas aeruginosa clinical and environmental isolates constitute a single population with high phenotypic diversity. BMC Genomics 15:318. doi: 10.1186/1471-216415-318

Guilbault, C., Saeed, Z., Downey, G. P., and Radzioch, D. (2007). Cystic fibrosis mouse models. Am. J. Respir. Cell Mol. Biol. 36, 1-7. doi: 10.1165/rcmb.20060184TR

Hassett, D. J., Ma, J. F., Elkins, J. G., McDermott, T. R., Ochsner, U. A., West, S. E., et al. (1999). Quorum sensing in Pseudomonas aeruginosa controls expression of catalase and superoxide dismutase genes and mediates biofilm susceptibility 
to hydrogen peroxide. Mol. Microbiol. 34, 1082-1093. doi: 10.1046/j.13652958.1999.01672.x

Hauser, A. R. (2009). The type III secretion system of Pseudomonas aeruginosa: infection by injection. Nat. Rev. Microbiol. 7, 654-665. doi: 10.1038/nrmicro 2199

Hazan, R., He, J., Xiao, G., Dekimpe, V., Apidianakis, Y., Lesic, B., et al. (2010). Homeostatic interplay between bacterial cell-cell signaling and iron in virulence. PLoS Pathog. 6:e1000810. doi: 10.1371/journal.ppat.1000810

Hentzer, M., Wu, H., Andersen, J. B., Riedel, K., Rasmussen, T. B., Bagge, N., et al. (2003). Attenuation of Pseudomonas aeruginosa virulence by quorum sensing inhibitors. EMBO J. 22, 3803-3815. doi: 10.1093/emboj/cdg366

Heurlier, K., Dénervaud, V., Haenni, M., Guy, L., Krishnapillai, V., and Haas, D. (2005). Quorum-sensing-negative (lasR) mutants of Pseudomonas aeruginosa avoid cell lysis and death. J. Bacteriol. 187, 4875-4883. doi: 10.1128/JB.187.14.4875-4883.2005

Hoffmann, N., Lee, B., Hentzer, M., Rasmussen, T. B., Song, Z., Johansen, H. K., et al. (2007). Azithromycin blocks quorum sensing and alginate polymer formation and increases the sensitivity to serum and stationary-growth-phase killing of Pseudomonas aeruginosa and attenuates chronic $P$. aeruginosa lung infection in Cftr(-/-) mice. Antimicrob. Agents Chemother. 51, 3677-3687. doi: 10.1128/AAC.01011-06

Hoffmann, N., Rasmussen, T. B., Jensen, P. Ø., Stub, C., Hentzer, M., Molin, S., et al. (2005). Novel mouse model of chronic Pseudomonas aeruginosa lung infection mimicking cystic fibrosis. Infect. Immun. 73, 2504-2514. doi: 10.1128/IAI.73.4.2504-2514.2005

Jakobsen, T. H., van Gennip, M., Phipps, R. K., Shanmugham, M. S., Christensen, L. D., Alhede, M., et al. (2011). Ajoene, a sulfur-rich molecule from garlic, inhibits genes controlled by quorum sensing. Antimicrob. Agents Chemother. 56, 2314-2325. doi: 10.1128/AAC.05919-11

Jimenez, P. N., Koch, G., Thompson, J. A., Xavier, K. B., Cool, R. H., and Quax, W. J. (2012). The multiple signaling systems regulating virulence in Pseudomonas aeruginosa. Microbiol. Mol. Biol. Rev. 76, 46-65. doi: 10.1128/MMBR.05007-11

Kalia, V. C., Wood, T. K., and Kumar, P. (2013). Evolution of resistance to quorumsensing inhibitors. Microb. Ecol. 68, 13-23. doi: 10.1007/s00248-013-0316-y

Kohler, T., Perron, G. G., Buckling, A., and van Delden, C. (2010). Quorum sensing inhibition selects for virulence and cooperation in Pseudomonas aeruginosa. PLoS Pathog. 6:e1000883. doi: 10.1371/journal.ppat.1000883

Kong, W., Liang, H., Shen, L., and Duan, K. (2009). [Regulation of type III secretion system by Rhl and PQS quorum sensing systems in Pseudomonas aeruginosa]. Wei Sheng Wu Xue Bao 49, 1158-1164. Available online at: http://journals.im. ac.cn/actamicrocn/ch/reader/view_abstract.aspx?file_no=909-5

Koul, S., Prakash, J., Mishra, A., and Kalia, V. C. (2016). Potential emergence of multi-quorum sensing inhibitor resistant (MQSIR) bacteria. Indian J. Microbiol. 56, 1-18. doi: 10.1007/s12088-015-0558-0

Laarman, A. J., Bardoel, B. W., Ruyken, M., Fernie, J., Milder, F. J., van Strijp, J. A., et al. (2013). Pseudomonas aeruginosa alkaline protease blocks complement activation via the classical and lectin pathways. J. Immunol. 188, 386-393. doi: 10.4049/jimmunol.1102162

Lee, D. G., Urbach, J. M., Wu, G., Liberati, N. T., Feinbaum, R. L., Miyata, S., et al. (2006). Genomic analysis reveals that Pseudomonas aeruginosa virulence is combinatorial. Genome Biol. 7:R90. doi: 10.1186/gb-2006-7-10-r90

Lee, J., and Zhang, L. (2015). The hierarchy quorum sensing network in Pseudomonas aeruginosa. Protein Cell 6, 26-41. doi: 10.1007/s13238-0140100-x

Lesic, B., Lépine, F., Déziel, E., Zhang, J., Zhang, Q., Padfield, K., et al. (2007). Inhibitors of pathogen intercellular signals as selective anti-infective compounds. PLoS Pathog. 3, 1229-1239. doi: 10.1371/journal.ppat.0030126

Maeda, T., García-Contreras, R., Pu, M., Sheng, L., Garcia, L. R., Tomás, M., et al. (2012). Quorum quenching quandary: resistance to antivirulence compounds. ISME J. 6, 493-501. doi: 10.1038/ismej.2011.122

Martinelli, D., Grossmann, G., Sequin, U., Brandl, H., and Bachofen, R. (2004). Effects of natural and chemically synthesized furanones on quorum sensing in Chromobacterium violaceum. BMC Microbiol. 4:25. doi: 10.1186/14712180-4-25

Mittal, R., Sharma, S., Chhibber, S., and Harjai, K. (2008). Iron dictates the virulence of Pseudomonas aeruginosa in urinary tract infections. J. Biomed. Sci. 15, 731-741. doi: 10.1007/s11373-008-9274-7
O’Loughlin, C. T., Miller, L. C., Siryaporn, A., Drescher, K., Semmelhack, M. F., and Bassler, B. L. (2013). A quorum-sensing inhibitor blocks Pseudomonas aeruginosa virulence and biofilm formation. Proc. Natl. Acad. Sci. U.S.A. 110, 17981-17986. doi: 10.1073/pnas.1316981110

Palmer, K. L., Aye, L. M., and Whiteley, M. (2007). Nutritional cues control Pseudomonas aeruginosa multicellular behavior in cystic fibrosis sputum. J. Bacteriol. 189, 8079-8087. doi: 10.1128/JB.01138-07

Patel, N. J., Zaborina, O., Wu, L., Wang, Y., Wolfgeher, D. J., Valuckaite, V., et al. (2007). Recognition of intestinal epithelial HIF-1alpha activation by Pseudomonas aeruginosa. Am. J. Physiol. Gastrointest. Liver Physiol. 292, G134G142. doi: 10.1152/ajpgi.00276.2006

Poole, K. (2011). Pseudomonas aeruginosa: resistance to the max. Front. Microbiol. 2:65. doi: 10.3389/fmicb.2011.00065

Rumbaugh, K. P., Griswold, J. A., and Hamood, A. N. (1999a). Contribution of the regulatory gene lasR to the pathogenesis of infection of burned mice. J. Burn Care Rehabil. 20, 42-49. doi: 10.1097/00004630-199901001-00008

Rumbaugh, K. P., Griswold, J. A., Iglewski, B. H., and Hamood, A. N. (1999b). Contribution of quorum sensing to the virulence of Pseudomonas aeruginosa in burn wound infections. Infect. Immun. 67, 5854-5862.

Saiman, L., Marshall, B. C., Mayer-Hamblett, N., Burns, J. L., Quittner, A. L., Cibene, D. A., et al. (2003). Azithromycin in patients with cystic fibrosis chronically infected with Pseudomonas aeruginosa: a randomized controlled trial. JAMA 290, 1749-1756. doi: 10.1001/jama.290.13.1749

Sana, T. G., Hachani, A., Bucior, I., Soscia, C., Garvis, S., Termine, E., et al. (2012). The second type VI secretion system of Pseudomonas aeruginosa strain PAO1 is regulated by quorum sensing and Fur and modulates internalization in epithelial cells. J. Biol. Chem. 287, 27095-27105. doi: 10.1074/jbc.M112.376368

Sandoz, K. M., Mitzimberg, S. M., and Schuster, M. (2007). Social cheating in Pseudomonas aeruginosa quorum sensing. Proc. Natl. Acad. Sci. U.S.A. 104, 15876-15881. doi: 10.1073/pnas.0705653104

Sarkisova, S., Patrauchan, M. A., Berglund, D., Nivens, D. E., and Franklin, M. J. (2005). Calcium-induced virulence factors associated with the extracellular matrix of mucoid Pseudomonas aeruginosa biofilms. J. Bacteriol. 187, 4327-4337. doi: 10.1128/JB.187.13.4327-4337.2005

Schuster, M., Lostroh, C. P., Ogi, T., and Greenberg, E. P. (2003). Identification, timing, and signal specificity of Pseudomonas aeruginosa quorumcontrolled genes: a transcriptome analysis. J. Bacteriol. 185, 2066-2079. doi: 10.1128/JB.185.7.2066-2079.2003

Sheng, L., Pu, M., Hegde, M., Zhang, Y., Jayaraman, A., and Wood, T. K. (2012). Interkingdom adenosine signal reduces Pseudomonas aeruginosa pathogenicity. Microb. Biotechnol. 5, 560-572. doi: 10.1111/j.1751-7915. 2012.00338.x

Smith, R. S., and Iglewski, B. H. (2003). Pseudomonas aeruginosa quorum sensing as a potential antimicrobial target. J. Clin. Invest. 112, 1460-1465. doi: 10.1172/JCI200320364

Sokol, P. A., and Woods, D. E. (1984). Relationship of iron and extracellular virulence factors to Pseudomonas aeruginosa lung infections. J. Med. Microbiol. 18, 125-133. doi: 10.1099/00222615-18-1-125

Sperandio, V., Li, C. C., and Kaper, J. B. (2002). Quorum-sensing Escherichia coli regulator A: a regulator of the LysR family involved in the regulation of the locus of enterocyte effacement pathogenicity island in enterohemorrhagic $E$. coli. Infect. Immun. 70, 3085-3093. doi: 10.1128/IAI.70.6.3085-3093.2002

Stover, C. K., Pham, X. Q., Erwin, A. L., Mizoguchi, S. D., Warrener, P., Hickey, M. J., et al. (2000). Complete genome sequence of Pseudomonas aeruginosa PAO1, an opportunistic pathogen. Nature 406, 959-964. doi: 10.1038/35023079

Tomás, M., Doumith, M., Warner, M., Turton, J. F., Beceiro, A., Bou, G., et al. (2010). Efflux pumps, OprD porin, AmpC beta-lactamase, and multiresistance in Pseudomonas aeruginosa isolates from cystic fibrosis patients. Antimicrob. Agents Chemother. 54, 2219-2224. doi: 10.1128/AAC.00816-09

Wagner, V. E., Bushnell, D., Passador, L., Brooks, A. I., and Iglewski, B. H. (2003). Microarray analysis of Pseudomonas aeruginosa quorum-sensing regulons: effects of growth phase and environment. J. Bacteriol. 185, 2080-2095. doi: 10.1128/JB.185.7.2080-2095.2003

Welsh, M. A., Eibergen, N. R., Moore, J. D., and Blackwell, H. E. (2015). Small molecule disruption of quorum sensing cross-regulation in Pseudomonas aeruginosa causes major and unexpected alterations to virulence phenotypes. J. Am. Chem. Soc. 137, 1510-1519. doi: 10.1021/ja5110798 
Wretlind, B., and Pavlovskis, O. R. (1983). Pseudomonas aeruginosa elastase and its role in pseudomonas infections. Rev. Infect. Dis. 5(Suppl. 5), S998-S1004. doi: 10.1093/clinids/5.supplement_5.s998

Wu, H., Song, Z., Hentzer, M., Andersen, J. B., Molin, S., Givskov, M., et al. (2004). Synthetic furanones inhibit quorum-sensing and enhance bacterial clearance in Pseudomonas aeruginosa lung infection in mice. J. Antimicrob. Chemother. 53, 1054-1061. doi: 10.1093/jac/ dkh223

Yang, Q., and Defoirdt, T. (2015). Quorum sensing positively regulates flagellar motility in pathogenic Vibrio harveyi. Environ. Microbiol. 17, 960-968. doi: 10.1111/1462-2920.12420

Zaborin, A., Romanowski, K., Gerdes, S., Holbrook, C., Lepine, F., Long, J., et al. (2009). Red death in Caenorhabditis elegans caused by Pseudomonas aeruginosa PAO1. Proc. Natl. Acad. Sci. U.S.A. 106, 6327-6332. doi: 10.1073/ pnas.0813199106
Zhu, J., Miller, M. B., Vance, R. E., Dziejman, M., Bassler, B. L., and Mekalanos, J. J. (2002). Quorum-sensing regulators control virulence gene expression in Vibrio cholerae. Proc. Natl. Acad. Sci. U.S.A. 99, 3129-3134. doi: 10.1073/pnas.052694299

Conflict of Interest Statement: The author declares that the research was conducted in the absence of any commercial or financial relationships that could be construed as a potential conflict of interest.

Copyright (c) 2016 Garcia-Contreras. This is an open-access article distributed under the terms of the Creative Commons Attribution License (CC BY). The use, distribution or reproduction in other forums is permitted, provided the original author(s) or licensor are credited and that the original publication in this journal is cited, in accordance with accepted academic practice. No use, distribution or reproduction is permitted which does not comply with these terms. 